\title{
Extractos vegetales: alternativa de control de Colaspis sp. (Coleoptera: Chrysomelidae) en plátano cv. Harton.
}

\author{
Plant extracts: alternative control Colaspis sp. \\ (Coleoptera: Chrysomelidae) in banana cv. Harton.
}

\author{
José Luís Barrera Violeth¹, Claudio Fernández Herrera², Karol Darío Pérez garcía*
}

Recibido para publicación: Mayo 29 de 2017 - Aceptado para publicación: Noviembre 23 de 2017

\begin{abstract}
RESUMEN
El banano, principal producto de exportación en Colombia, presenta muchos retos técnicos como el control de la tortuguilla (Colaspis sp.), causante del 33\% de pérdidas económicas; dado que los estándares de calidad no permiten la presencia de residuos químicos presentes en los frutos, se evaluó en un cultivo comercial, del municipio de los Córdobas, el efecto de extractos vegetales en el manejo de la tortuguilla. Se evaluó la severidad del ataque a partir del número de roeduras en frutos y la incidencia en racimos y frutos tratados. Los extractos utilizados fueron salvia Salvia officinalis L., anamú Petiveria alliacea L., albahaca Ocimum basilicum L., ajo Allium sativum L. y ají picante Capsicum annuum asperjados con una bomba tipo jardinera sobre bolsas comerciales de polietileno; dejándose expuestas durante una hora para asegurar la adherencia del producto. Se estableció un diseño completamente al azar con siete tratamientos, tres repeticiones, un testigo relativo (bolsa sin extracto vegetal) y un testigo absoluto (racimos sin embolsar); registrando la severidad del daño causado. Los resultados mostraron que los frutos provenientes de racimos embolsados presentaron menor severidad que los frutos no embolsados y los tratamientos constituidos por bolsas impregnadas con extracto de ajo presentaron menor incidencia y grado de severidad, mostrando una disminución del daño en un $98 \%$. Concluyendo que el extracto de ajó fue el producto repelente que mejor acción mostro al daño de fruto. Los tratamientos donde se aplicó Albaca, Ají, Salvia y Anamú se pudieron determinar una mayor incidencia y daño de fruto.
\end{abstract}

Palabras clave: Extractos, severidad, daño, calidad, embolsados, Colaspis sp

\begin{abstract}
Bananas, the main export goods of Colombia, present various technical challenges such as the control of the turtle beetle (Colaspis sp.), which causes 33\% of economic losses. Since quality standards do not allow chemical residues to be present in the fruits, this study evaluated the effect of plant extracts for the management of the turtle beetle in a commercial crop from the municipality of Los Córdobas. The severity of the attack was evaluated from the number of gnawing in fruit and the incidence in treated bunches and fruits. The extracts used were salvia Salvia officinalis L., anamú Petiveria alliacea L., basil Ocimum basilicum L., garlic Allium sativum L. and chili pepper Capsicum annuum sprinkled with a planter type bomb on commercial polyethylene bags; leaving exposed for one hour to ensure adhesion. A design was completely randomized with seven treatments, three repetitions, a relative witness (bag without plant extract) and an absolute control (unbagged bunches) recording the severity of the damage. The results showed that the fruits from bagged clusters had lower severity than the fruits of not bagged clusters. Treatments consisting of bags impregnated with garlic extract had lower incidence and severity, a decrease of $98 \%$ in damage. It could be concluded that the garlic extract repellent showed the best results to prevent fruit damage. Treatments where Albaca, Chili Pepper, Salvia and Anamú ware applied showed higher incidence and fruit damage.
\end{abstract}

Key words: Extracts, severity, damage, quality, pocketed, Colaspis sp.

\footnotetext{
${ }^{1}$ M.Sc. Docente. Facultad de Ciencias Agrícolas, Universidad de Córdoba. E-mail: jbarrera11@correo.unicordoba.edu.co

${ }^{2}$ M.Sc. Docente. Facultad de Ciencias Agrícolas, Universidad de Córdoba. E-mail: claudiofernandezherrera@gmail.com

$3^{3 *}$ M.Sc. Docente. Facultad de Ciencias Agrícolas. Universidad de Córdoba. Montería, Córdoba, Colombia. A. A. 354 . Tel. +57(4) 7860255

karolperez0414@hotmail.com
} 


\section{INTRODUCCIÓN}

En Colombia existen actualmente más 348.000 ha cultivadas con plátano, ocupando el tercer lugar de los sistemas perennes, después del café y caña (DANE, 2015). La producción en 2008 alcanzó 2,8 millones de ton., de las cuales se exportaron el $3,6 \%$, cerca del $1 \%$ se destinó a la agroindustria y se estimaron pérdidas equivalentes al $10 \%$ de la producción, el resto se consumió en los hogares rurales y urbanos del país (Ruiz y Ureña, 2009).

La tendencia actual de comercialización del plátano es hacia los mercados especializados, la cual se realiza en la presentación de manos y frutos; por tanto, las dimensiones de los dedos determinan el precio de venta y su aceptación por estos mercados (CCl, 2000).

La incidencia de plagas y enfermedades ocasiona pérdidas que alcanzan una tercera parte de la producción la cual debe destruirse, lo que conlleva al estudio de nuevas vías para su control. Por otro lado, son numerosos los insectos asociados al cultivo de banano que pueden llegar a causar pérdidas económicas cuando se incrementa su presencia y adquieren el estatus de plaga (Medina, 2001).

Normalmente las poblaciones altas son provocadas por alteración de la relación entre la plaga y su enemigo natural, asociados generalmente a desequilibrios ecológicos por cambios climáticos o a una mala práctica de manejo, las cuales en general, afectan negativamente a sus controladores biológicos (Hilje, 1994).

Esta estrategia ecoeficiente en el manejo de artropodos plagas se viene investigando en el uso de metabolitos secundarios (terpenos, lignanos, alcaloides, esteroides y ácidos grasos) tienen un rol importante en el mecanismo defensivo de las plantas (Polack et al., 2005); por tanto, en los últimos años, se está retornando al uso de las plantas como fuente de pesticidas más seguros para el medio ambiente y la salud humana (Mansaray, 2000; Ottaway, 2001).

En las regiones productoras de plátano y banano para la exportación, Colaspis sp. pueden considerarse como una de las plagas del fruto de mayor importancia económica, puesto que se alimenta ocasional o frecuentemente del fruto recién emergido hasta tres semanas de edad afectando considerablemente su presentación y la cantidad de fruta exportable (Dane, 2015; Belalcazar, 1991).

En áreas con daño en hoja sin desplegarse facilita su identificación e inclusive la estimación de su población ya que el daño en el follaje se caracteriza por mostrar agujeros de forma irregular que tiende a ser más grandes en el margen derecho de la hoja. Los adultos se esconden durante el día en los colinos, por ser una plaga de hábitos nocturnos, y se localizan en las axilas de las hojas; y los niveles de daño observados en la fruta pueden llegar hasta un 100\%, sino se realiza algún método de manejo (AUGURA, 2009).

En la producción del plátano, la labor del embolse es fundamental para proteger el racimo de los efectos abrasivos causados por los productos químicos, daños relacionados con raspones, quemaduras en el epicarpio por el roce de las hojas dobladas, puntales, proceso de corte y acarreo y principalmente del ataque de insectos plagas del fruto como Colaspis sp. sin embargo, esto no ha sido suficiente, requiriendo de la utilización de insecticidas para proteger eficientemente al racimo del ataque de plagas (Ostmark, 1975).

Los biopesticidas de origen vegetal vienen implementándose con éxito, en el manejo de artropodos plagas que además, de controlar la plaga y los daños del cultivo, proteje el medio ambiente de la contaminación por pesticidas (Mazid et al., 2011). 
Asimismo, la presión social ha incentivado el uso de sustancias naturales para el control de plagas y enfermedades en vegetales, a tal punto que muchos productos de exportación deben adecuarse a las condiciones de cultivo orgánico (Aquino y Stauffer, 2000). Por tanto, es necesario avanzar en la investigación del potencial de nuestra flora para el desarrollo de nuevos subproductos con poder insecticida y/o repelente en cosecha de frutas y hortalizas.

Todo lo expuesto ha motivado la búsqueda de integración de medios alternativos siendo los productos orgánicos una opción aceptable. Entre estos se incluye el uso de barreras físicas, insecticidas vegetales, jabones agrícolas, hongos entomopatogenos, enemigos naturales e insecticidas sintéticos de acción selectiva. La utilización armónica de estas estrategias constituye una herramienta importante en los programas de manejo integrado de plagas. Por esta razón el objetivo de esta investigación consistió en determinar la efectividad de los extractos vegetales en el manejo de Colaspis sp. en plátano.

\section{MATERIALES Y MÉTODOS}

El estudio se desarrollo en el corregimiento La Salada, ubicado en el municipio Los Córdobas. Departamento de Córdoba, Colombia, correspondiente a las coordenadas geográficas $8^{\circ} 54^{`}$ de latitud norte y $75^{\circ} 21^{`}$ de longitud oeste con respecto al meridiano de Greenwich, con temperatura promedio de $28{ }^{\circ} \mathrm{C}$, humedad relativa del $86 \%$ y precipitación promedio anual de $1.300 \mathrm{~mm}$, ecológicamente corresponde a la denominación de bosque seco tropical.

Los extractos utilizados fueron hidrolatos que se obtuvieron en el laboratorio de productos naturales de la Universidad de Córdoba, a partir de hojas sanas humedeciendo con de solvente (etanol o agua). Los extractos se obtuvieron mediante el método de percolación a partir del material macerado en un recipiente metálico y con un mortero de loza, una vez macerado se dejaron fermentando por 72 horas en agua estéril, en una relación de 4 litros de agua por $1 \mathrm{~kg}$ de hoja, y finalmente se extrajo el liquido del fermentado, se filtró y se envasó en frascos de vidrio color ámbar (para evitar la fotolisis), rotulados, y guardados a temperatura de $28{ }^{\circ} \mathrm{C} \pm 3{ }^{\circ} \mathrm{C}$ hasta el día a ser utilizadas en los bioensayos (lannacone y Lamas, 2003a). Para obtener mayor cantidad de principios activos se recirculó el extracto. Posteriormente se sometió al rotoevaporador con el objetivo de disminuir el exceso de solvente y obtener un extracto más puro.

Con el propósito de evaluar la eficiencia de los extractos empleados para el control de Colaspis sp. se establecieron cincos tratamientos que consistieron en extractos vegetales que se obtuvieron del jardín medicinal de la Universidad de Córdoba tales como; salvia Salvia officinalis L. (Lamiáceas); anamú Petiveria alliacea L. (Fitolacáceas); albahaca Ocimum basilicum L. (Lamiáceas); el ajo Allium sativum L. (Liliáceas) y ají picante Capsicum annuum (Solanaceae); se compró en almacenes de cadena. Los extractos se aplicaron asperjándolos con una bomba tipo jardinera de 5 litros sobre las bolsas comerciales de polietileno aplicándosele aproximadamente $50 \mathrm{ml}$ de la solución acuosa (extracto + agua) (Tabla 1). Estas bolsas actuaban como protección de racimos de acuerdo a los tratamientos evaluados; y se dejaron expuestas durante una hora para su secado bajo techo, con el fin de asegurar la adherencia del producto a la bolsa de polietileno de baja densidad.

Tabla 1. Extractos de plantas evaluadas para el control de Colaspis sp. en frutos de plátano (Musa AAB Simmonds) Cv.

\begin{tabular}{ccccc}
\hline Tratamientos & Embolse & $\begin{array}{c}\text { Extracto vegetal } \\
\text { Nombre común }\end{array}$ & $\begin{array}{c}\text { Extracto vegetal } \\
\text { Nombre común }\end{array}$ & $\begin{array}{c}\text { Parte } \\
\text { utilizada }\end{array}$ \\
\hline 1 & $\mathrm{Si}$ & Salvia & (Salvia officinalis) & Hojas \\
2 & $\mathrm{Si}$ & Anamú & (Petiveria alliacea) & Hojas \\
3 & $\mathrm{Si}$ & Albahaca & (Ocimum basilicum) & Hojas \\
4 & $\mathrm{Si}$ & Ajo & (Allium sativum) & Hojas \\
5 & $\mathrm{Si}$ & Ají picante & (Capsicum sp.) & Hojas \\
6 (Testigo relativo) & $\mathrm{Si}$ & --------- & --------- & ---- \\
7 (Testigo absoluto) & $\mathrm{No}$ & ------ & ---- \\
\hline
\end{tabular}


Para su fase de campo se estableció un diseño completamente al azar, con siete tratamientos y tres repeticiones. La población estuvo constituida por 480 plantas de plátano tipo Hartón sembradas a 2,7 m x 2,7 m en triangulo. Los tratamientos correspondieron a la impregnación de extractos vegetales con propiedades repelentes $y / o$ insecticidas en las bolsas usadas en la protección de los racimos.

Los respectivos embolses se hicieron en el estado prematuro, es decir, cuando la inflorescencia ha abierto máximo dos brácteas y posteriormente para la toma de los datos sobre la evaluación de Colaspis sp. en los racimos se realizó en el momento de la cosecha cuando el cultivo alcanzo la edad de 12 meses, donde se registraba la influencia del insecto a partir de las lesiones superficiales en forma de serpentina que dejaba sobre la cáscara del fruto y preferiblemente entre las aristas o filos del fruto.

Se evaluó la severidad del ataque de la plaga a partir del número de roeduras presentada por fruto de acuerdo a cada tratamiento y el porcentaje de incidencia se estimó mediante: \%incidencia $=$ [numero de frutos afectados/numero de frutos totales] x 100 .

La toma de datos de la plaga en estudio se hizo en cuatro muestreos a partir de los 70 días después de emitida la inflorescencia, sobre los racimos que presentaban las características de madures comercial con fines de exportación, y se evaluó la totalidad de los frutos por racimo, para este proceso se realizo la cosecha y se procedió hacer la evaluación correspondiente durante la mayor incidencia de la plaga y cosecha de frutos. Para el análisis de datos de campo se utilizó el progama estadístico SAS versión 6.12 (Ray 1982). Se realizó el análisis de varianza convencional y la diferencia de los tratamientos mediante la prueba de comparación de medias de rango múltiple de Tukey $(\mathrm{P}<0,05)$.

\section{RESULTADOS Y DISCUSIÓN}

Los resultados sobre la aplicaciones de los extractos vegetales que se emplearon en los cuatros muestreos, mostro en el análisis de varianza diferencias significativas entre los tratamientos $(\mathrm{P}<0,05)$, dondelostratamientosque fueron sometidos a la aplicación de los extractos muestran una incidencia en función del sistema de aplicación y difirieron estadísticamente del tratamiento testigo quien presenta porcentajes de incidencia de Colaspis significativamente superiores al resto de tratamientos ensayados.

Esto puede estar influenciado por la cantidad de los componentes activos en el extracto final y particularmente del origen de la planta; también se le suma el estado de desarrollo de la planta en la cosecha, el proceso de secado y las técnicas de almacenamiento; todo esto pueden incidir en la concentración de los ingredientes activos.

Los valores de los análisis de varianza para la incidencia de insecto para cada uno de los extractos analizados también ponen de manifiesto el efecto repelente de éstos; como también lo expresa Munakata (1977) quien prepondera la importancia de la actividad antialimentaria y hace mención a que soluciones acetónicas al 5\% de extractos de hojas de varias especies de las familias Araceae, Compositae, Euphorbiaceae, Labiatae, Lauraceae, Leguminosae y Verbenaceae poseen dicha cualidad.

Por su parte diversos autores estudiaron los efectos de extractos de plantas sobre el comportamiento alimentario de T. castaneum y otros gorgojos de granos almacenados (Laudani and Swank 1954), (Jilani and Malik 1973), (Jilani et al., 1988), (Jilani and Su, 1983). Talukder and Howse (1993) observaron efectos repelentes y actividad tóxica de extractos de la planta tropical. Si bien el análisis de la varianza indica que hay diferencias significativas entre ellos $(P>0,05)$, aquí se observa el comportamiento superior de 
los extractos comparados con sus respectivos testigos sin tratar. Es por esto que los tratamientos salvia ( $S$. officinalis), albahaca (O. basilicum) y ají picante $(C$. annuum) no difieren entre ellos. Esto se debe a compuestos de las plantas pueden ejercer un efecto antialimentario y a su vez llegar a afectar actividades biológicas o alteraciones hormonales; datos que se asemejan a los reportados Ducrot (2004) quien afirma que muchas veces puede hasta llevar a la muerte a los insectos.

Peropara determinarlo sería necesario identificar y aislar por diversos métodos analíticos las moléculas que componen este tipo de extracto, para determinar cuál o cuáles son los que generan los efectos insecticida-antialimentario y si la acción se debe a un efecto sinérgico entre los metabolitos presentes en los extracto.

Se pudo determinar que Colaspis por presentar hábitos nocturnos, su mayor actividad de alimentación se registra en horas de la noche $y$ en condiciones de baja intensidad solar; estos resultados se pueden confirmar en lo observado por Gonzaga (1999; 2006); GualánVega (2013), quienes afirman que durante el día se encuentra refugiados e inmóviles en los hijos de aguja, la planta adulta del banano (hoja bandera), las brácteas de la inflorescencia ya desprendidas y enrolladas, en las pequeñas grietas del suelo, entre los dedos tiernos cuando realizan labor de deshije; de donde sale después a realizar sus procesos de alimentación.

Todo parece indicar que la bolsa con los extractos vegetales incorporado, presentaron el más bajo efecto insecticida, debido a que el agua extrae solo compuestos de alta polaridad, como glucósidos (Cortés, 2008), la mayoría de los cuales tienen una baja actividad biológica (Ducrot, 2004). Es por ello que estos extractos presentaron una mejor acción sobre Colaspis sp. a manera de un efecto más de repelente, de tal manera que las poblaciones del insecto tienden a aumentar o, al menos a permanecer estables.
Caso contrario a los resultados obtenidos en el ensayo donde se impregnaron las bolsas con extracto de ajo, quien presento la menor severidad con 0,5 roeduras/dedo; debido a la alta concentración que se utilizo de ajos el cual estaba representado en un 95\%, lo que explica la mayor efectividad de este producto en la reducción de las poblaciones del insecto por lo cual se podría considerar como una dosis adecuada de la preparación del extracto para controlar adultos de Colaspis sp. Sin embargo es bueno resaltar que los insectos se vuelven resistentes a los pesticidas sintéticos, generando el temor de que el uso a gran escala de insecticidas a base de ajo puede conducir a la resistencia del fitófago especialmente de Colaspis, aunque esto no se ha expresado; si se ha demostrado que proporcionan protección a las planta a largo plazo contra el ataque de plagas.

También los resultados muestran que en los insectos con mayor movilidad como Colaspis sp., el producto repelente actúa según lo esperado debido a sus productos azufrados como Alicina, Alina, Cicloide de Alicina y Disulfuro de Dialil con propiedades repelentes y que están presentes en el ajo, es por esto que han resultado eficaces en la reducción del nivel de daño de insectos según Special Nutrients (1996a), donde afirma que el insecto se dirige a un punto opuesto al estímulo, mientras que en los ápteros la actividad repelente se manifiesta más tarde.

Estos resultados se asemejan a los publicados por Special Nutrients (1996b), mediante el uso de repelentes derivados de ajo para el control de áfidos en el cultivo de papa orgánico y también sobre la eficacia en la reducción del nivel de daño por insectos en algodón. Dentro de estos resultados cabe mencionar que su modo de acción no mata huevecillos como tampoco larvas ya que estos estados no se evidenciaronacciónalgunaencampo, solamente repele a los adultos dentro del cultivo por lo que normalmente no da lugar a un porcentaje 
de mortalidad debido a que no presenta un alto poder insecticida. Consecuentemente, los procesos de alimentación de adultos de Colaspis sp. no fueron tampoco superior. Pero si puede dar lugar a una posterior reducción sustancial del que se apareen estos insectos, de modo que la siguiente generación se puede ver reducida por debajo del nivel de daño.

Al analizar estos resultados, concuerdan con los reportados por Rodríguez (1998) quien para el control de poblaciones de mosquita blanca y pulgones utilizo de 10-12 dientes de ajo picados y machacados y seis chiles picantes (Capsicum frutescens), mezclados en cuatro litros de agua, donde previamente se disolvió un cuarto de jabón de pasta.

En otra investigación realizada Rodríguez (1998) uso el extracto de ajo para controlar plagas de minador de las hojas de los cítricos (Phyllocnistis citrella) sobre árboles jóvenes, utilizaron 10 árboles con tres repeticiones, a un volumen de 15 gramos en 200 ml obteniendo como resultado que el promedio de larvas bajó en un $76,8 \%$, siendo el de mayor efectividad comparado a los efectos de la pimienta (Pimienta dioica) y el acuyo (Piper auritum).

La impregnación de bolsas con extracto de albahaca, ají picante, salvia y anamú se presento una mayor severidad de roeduras/dedo de 2,8 - 2,9 - 3,0 y 4,6 respectivamente (Figura 1). Comparando estos resultados podemos decir que coinciden con reportados por Rivera et al. (2003) en cultivos de papa en Cuba, quienes manifiestan que el uso de extractos de ajo ha registrado efectos positivos en el control de artrópodos como el áfido Carolinaia cyperi en aplicaciones con una frecuencia semanal, cuyas aplicaciones contenían 43 g de ajo (dientes secos) e igual cantidad de tabaco (hojas secas) mezcladas en $3 \mathrm{~L}$ de agua, se pusieron en ebullición 15 min. luego se dejó reposar 17 h, se filtró por gasa y se llevó al volumen de 3 L. Por su parte Ho et al. (1996), mostro mediante investigaciones y pruebas de toxicidad en contra de los huevos, larvas y adultos de Tribolium castaneum, adultos de Sitophilus zeamais, y huevos de Z. cutaneum, donde la mortalidad de estos insectos aumentó cuando se incremento la concentración de aceite con ajo en cultivos de arroz y trigo, afirmando que la destrucción completa de los huevos se logra a 4,4 $\mathrm{mg} \mathrm{cm}^{2}$, utilizando en el bioensayo papel filtro de impregnación.

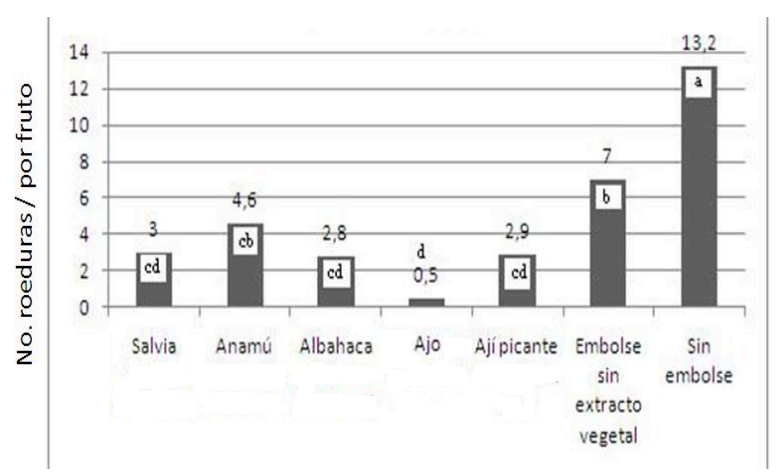

Medias con letras iguales no difieren significativamente, según Tukey $(\mathrm{P}<0,05)$.

Figura 1. Efecto de extractos vegetales en la protección de los racimos pare el control de Colaspis sp. en plátano cv. Hartón

En cultivos de tomate bajo cubierta Rodríguez (1993) y Redepapa (2000), demostraron la efectividad del extracto de ajo sobre mosca blanca Bemisia tabaci (Hemiptera: Aleyrodidae) demostrando que este extracto no produce mortalidad pero si acción repelente.

De acuerdo al porcentaje de incidencia para frutos dañados que fueron tratado con ajo presentó el menor porcentaje de afectación con un $2 \%$ ya que no se evidencio daño en la superficie de la cáscara, tal como se evidenció anteriormente en la figura 2, que se expresa los efectos de extractos vegetales, en donde no existe roeduras en fruta tierna (1-20 días de edad).

Los resultados concuerdan con los reportados por Carrero y Lizarazo (2006) quienes estudiaron el efecto de extractos en diferentes dosis y encontraron para aplicarlas a las larvas (1.000 


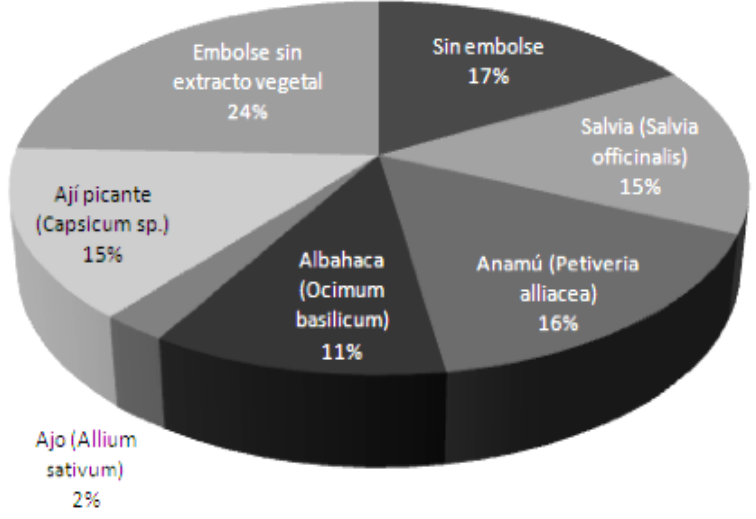

Figura 2. Efecto de extractos vegetales (\%) en la protección de frutos de plátano cv. Hrtón afectados por Colaspis sp.

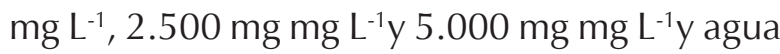
como testigo absoluto). A estas soluciones se les agregó coadyuvante $\left(2 \mathrm{~mL} \mathrm{~L}^{-1}\right)$; produciendo un efecto antialimentario e insecticida tipo know down de importancia económica sobre larvas de Spodoptera frugiperda criadas en condiciones de laboratorio.

Mientras que el testigo (Sin embolse sin extracto vegetal presento un daño de un $24 \%$ de frutos dañados los cuales presentaban cicatrices superficiales con halos acuosos, dañando por completo la presentación de la fruta lo cual disminuye la cantidad de fruta (Figura 2), seguido por un $17 \%$ de los frutos donde solo se embolsó el racimo.

Estos resultados indican un uso potencial de los extractos más destacados de las plantas estudiadas como se pudo evidenciar en la grafica anterior, o de productos derivados de ellas, en la protección del cultivo; para ello se deberán seguir realizando estudios tendientes a identificar, separar y cuantificar los compuestos activos responsables de la actividad repelente. Vásquez (2005), reporto experimentos para controlar el gusano cogollero del maíz, mostrando que el tratamiento de ajo mas aceite mineral y jabón constituyo la mejor alternativa para el control de dicha plaga reduciendo así la incidencia de la plaga. Finalmente, los extractos vegetales de salvia
S. officinalis, anamú $P$. alliacea, albahaca O. basilicum y ají picante $C$. annuum, no fueron consideradas altamente promisorias ya que se expresó desde un 45 hasta el $65 \%$ de daño en frutos de plátanos ya que al comer realizan raspaduras y agujeros a lo largo de los frutos como a través de la superficie plana de los dedos tiernos.

Esto se debe a que el uso de repelentes a base de estos extractos en cuanto a niveles de población del insecto resultan poco eficientes y tal vez dependa de la preparación la cual incide a que tenga una respuesta menor en esos parámetros que un producto preparado industrialmente. Por ello, se debe generar investigaciones posteriores que modifiquen la técnica de preparación y de aplicación en el producto para poder generar una alternativa apropiada para el agricultor orgánico.

\section{CONCLUSSIONS}

En la presente investigación se demostró que la aplicación de los extractos vegetales puede llegar a controlar insectos plaga utilizando plantas; por tal motivo el estudio de la acción biológica de más especies vegetales en diferentes plagas agrícolas puede ser una herramienta en el futuro para disminuir la aplicación de insumos de síntesis química.

El uso de repelentes a base de ajo resultó en cuanto a niveles de población de Colaspissp. y rendimiento mejor que el testigo sin aplicación, pero el extracto tiene respuestas menores en cuanto a la acción de insecticida. El extracto que produjo mejor respuesta repelente a la acción del daño de fruto a causa del Colaspis sp. fue el cual se aplico extracto de ajó. En los tratamientos donde se aplico Albaca, Ají, Salvia y Anamú se pudo determinar una mayor incidencia de insecto mostrando daño de fruto; además no mostraron diferencias significativas entre si, por lo que dichos productos no fueron lo suficientemente 
eficaces para el control de Colaspis sp.

Aunque el embolse produce un barrera física para que Colaspis sp. no dañe el fruto, este logra penetrar dentro de la bolsa ocasionando daño físico del 5\%. El no embolse y no aplicación de extracto de ajo, producen un daño total de los dedos alimentándose de la corteza de los frutos tiernos, haciendo roeduras alargadas de un milímetro de profundidad entre las aristas del fruto de plátano afectando así la calidad del fruto.

Los extractos de plantas Albahaca, Ají, Salvia y Anamú mostraron control de la plaga en comparación con el testigo, siendo el ajo el más eficiente. Sin embargo, es conveniente utilizar metodologías que determinen la eficiencia biológica del ajo en una acción más de mortalidad que de efectos repelentes sobre la plaga.

\section{REFERENCES}

Aquino, A. y Stauffer, A. 2000. Selección de extractos vegetales con efecto fungicida y bactericida. Rev Ciencia Tecnol. (Py) vol. $1 \mathrm{~N}^{\circ}$ 2. pp 29-30. http// www.newton. cnc.una.py/ resource-1006/2000v1n204pdf). [17 marzo 2016].

AUGURA (Asociación de Bananeros de Colombia). 2009. Guía para la Identificación y manejo integrado de plagas en banano y plátano Magdalena y Urabá. ISBN 978-958-99167-0-4. 64p.

ASIAVA-ICA-Ministerio de Agricultura y Desarrollo Rural. (2012). Monitoreo, evaluación, manejo y control de los principales problemas fitosanitarios del plátano, Musa paradisiaca y Banano, Musa sapientum, en zonas productoras del Valle del Cauca afectadas por la ola invernal.

Belalcazar C., Valencia J. y Lozada J. 1991. La planta y el fruto. En: Belalcazar, S (ed) Manual de Asistencia Técnica No. 50 ICA, El cultivo del plátano en el trópico. Armenia, Quindío, INIBAP. Feriva, Cali, pp.45-89,376. .
Dane, (Departamento Administrativo Nacional de Estadística). 2015. Comunicado de prensa. 3er Censo Nacional Agropecuario. Cód.: DIE-020-PD-01-r6_ v2. $10^{\circ}$ entrega.

Ducrot, P. 2004. Contribución de la química al conocimiento de la actividad biopesticida de los productos naturales de origen vegetal. En: Regnault-Roger, C., B.J.R. Philogene y C. Vincent (eds.). Biopesticidas de origen vegetal. Ediciones Mundi-Prensa, Madrid. 337 p.

Carrero, R. y Lizarazo, K. 2006. Evaluación de los efectos de extractos vegetales obtenidos a partir de Barbasco (Polygonum hydropiperoides), Hierba mora (Solanum nigrum) y Carbonero (Calliandra pittieri) sobre larvas deSpodoptera frugiperda (JE. SMITH) en segundo instar, bajo condiciones controladas. Trabajo de grado. Facultad de Ciencias Agropecuarias, Universidad de Cundinamarca, Fusagasugá. 75 p.

Corporación Colombiana Internacional (CCI) 2001. Acuerdo de competitividad de la cadena productiva del plátano en Colombia. COLECCION DOCUMENTOS IICA SERIE COMPETITIVIDAD No.18. Secretaría Técnica, Bogotá.

Cortés, D. 2008. Curso "Estructuras de moléculas naturales de interés terapéutico". Facultad de Farmacología, Universidad de Valencia, España. 12 p.

Gonzaga, V. 1999. Seminario: plagas del banano. Universidad Técnica de Machala. Machala, Ecuador. P. 18-19.

Gonzaga, V. 2006. Plagas del banano, reconocimiento, daños que causan, monitoreo y control. Monografía de la Cátedra de la cátedra de Entomología Económica. Universidad de Machala. 20p.

Gualán-Vega, J. 2013. Ciclo Biológico y Hábitos de Colaspis submetállica Jacoby, (COL: Chrysomelidae). Tesis Ing. Agr. Machala, Ecuador. Universidad Técnica de Machala, Facultad de Ciencias Agropecuarias, Escuela de Ingeniería Agronómica. 27p. 
Hilje, L. 1994. El manejo integrado de plagas como noción y estrategia para enfrentar los problemas de plagas. In Lecturas sobre manejo integrado de plagas. Eds. L. Hilje, O. Arboleda. Costa Rica, Programa de Agricultura Tropical Sostenible, Area de Fitoprotección. (237) pp. 1-23.

Ho, S., Koh, L., Ma, Y., Huang, Y. and Sim, K. 1996. The oil of garlic, Allium sativum L. (Amaryllidaceae), as a potential grain protectant against Tribolium castaneum (Herbst) and Sitophilus zeamais Motsch. Postharvest Biology and Technology 9 (1) 41-48. https://doi.org/10.1016/09255214(96)00018-X

lannacone, J. y Lamas, G. 2003a. Plantas biocidas usadas en el control de la polilla de la papa, Phthorimaea operculella (Zeller) (Lepidoptera: Gelechiidae). Rev. per. Ent. 43: 79-87.

Jilani, G. and Malik, M. 1973. Studies on neem plant as repellent against stored grain insects. Pak. J. Sci. Ind. Res. 16: 251-254.

Jilani, G. and Su, H. 1983. Laboratory studies on several plants materials as insect repellents for protection of cereal grains. Journal of Economic Entomology, 76(1): 154-157. https://doi.org/10.1093/ jee/76.1.154

Jilani, G., Saxena, R. and Rueda, B. 1988. Repellent and growth-Inhibiting effects of turmeric oil, sweetflag oil, neem oil and "Margosan-O" on red beetle (Coleoptera: Tenebrionidae). Journal of Economic Entomology, 81(4): 1226 -1230. https:// doi.org/10.1093/jee/81.4.1226

Laudani, H. and Swank, G. 1954. Laboratory apparatus for determmig repellency of pyrethrum when applied to grain. Journal of Economic Entomology, 47(6): 1104-1107. https://doi.org/10.1093/ jee/47.6.1104

Mansaray, M. 2000. Herbal remedies food or medicine?. Chem. Ind. 20(16), 677-678.

Mazid, S., Kalita, J. and Rajkhowa, R. 2011. A review on the use of biopesticides in insect pest management. International Journal of Science and Advanced Technology (ISSN 2221-8386). Volume 1 No 7. Pp 169-178.
Medina, N. 2001. Uso de extractos botánicos en control de plagas y enfermedades. Avances en el fomento de productos fitosanitarios no sintéticos. Manejo Integrado de Plagas (Costa Rica). 59, 7677.

Munakata, K. 1977. Insect antifeedants of Spodoptera litura in plants. En: Host plant resistance to pests. Ed. P.A. Hedin. American Chemical Society. Symposium Series 62 , Washington.

Ostmark, H. 1975. Banana pests in the genus Colaspis including description of a new species (Coleoptera: Chrysomelidae). Fla. Ent. 58(1):1-8. https://doi. org/10.2307/3493857

Ottaway, P. 2001. The roots of a health diet. Chem. Ind. 22, 42-44.

Polack, L. Silvestre, C. y Vega, C. 2005. Evaluación de un repelente basado en extracto de ajo en el control de la Mosca Blanca de los invernáculos, Trialeurodes vaporariorum, en cultivos de tomate bajo cubierta. http://www.inta.gov.ar/ sanpedro/info/doc/2005/ap_0501.htm. [15 Marzo 2016].

Ray, A. 1982. SAS User's guide. Statisties, SAS Institute Inc. Cary, N. C. 584 p.

Redepapa. 2000. Plantas insecticidas y plantas vivas como repelentes. Disponible en http://www.redepapa.org/ plantasinsecticidas.pdf . Acceso 10 de Mayo de 2014.

Rivera, A., Carballo, C., Milanés, M., Ramos, S. y Orama, R. 2003. Efecto de plaguicidas de origen botánico sobre el áfido Carolinaia cyperi Ainslie. Rev Cubana Plant Med. 8 (3): En linea : http://scielo.sld.cu/scielo.php?script=sci_ 